\title{
Monitoring Curing of Quasi-Isotropic Carbon Fiber/Epoxy Composite Using Fiber Bragg Grating Sensors
}

\author{
Chen-Tung Wang, Tsung-Han Hsieh, ${ }^{1}$ Chiu-Chen Chien, and Chia-Chin Chiang* \\ Department of Mechanical Engineering, National Kaohsiung University of Applied Sciences, \\ 415 Chien Kung Road, Kaohsiung 807, Taiwan \\ ${ }^{1}$ Department of Mold and Die Engineering, National Kaohsiung University of Applied Sciences, \\ 415 Chien Kung Road, Kaohsiung 807, Taiwan
}

(Received August 31, 2015; accepted February 4, 2016)

Keywords: optical fiber sensor, fiber Bragg grating, composite material, monitoring curing

In this study, four fiber Bragg grating (FBG) sensors were embedded into a quasi-isotropic carbon fiber/epoxy composite material to monitor curing. The spectra of the FBG sensors were shifted and deformed during the curing process. This phenomenon is caused by residual strain during composite curing. We monitored the spectral changes of the embedded FBG sensors in different laminate layers to measure the residual strain during curing. According to the experimental results, the curing residual strain of the composite was generated first from the central layer. The maximum axial and lateral residual strains measured by the FBG sensors were -456.7 and $1234.6 \mu \varepsilon$, respectively. The FBG sensors have advantages for use in the stress-strain sensing of composites.

\section{Introduction}

Composite materials have been widely applied in industry because of their specific strength, stiffness, and corrosion resistance. During the curing process for composite materials, residual strain is the factor most relevant to the quality of the composite product. Hence, the monitoring of curing is an important issue in the manufacturing of composite materials. Recently, the use of optical fiber Bragg gratings (FBGs) in curing monitoring has been widely studied. ${ }^{(1,2)}$ FBG sensors are small and compatible with common polymeric materials, and thus can be easily embedded close to the internal sensing site in a composite structure without introducing significant defects.

In 2009, Mulle et al..$^{(3)}$ embedded FBG with $\left[0^{\circ}\right]_{8}$ laminate in a carbon fiber/epoxy laminate composite. Change in the strain of the FBG in laminates of different thicknesses were investigated as a means of monitoring the curing process. It could be observed that the FBG was compressed in response to strain during the curing process as the temperature rose, presumably because the stickiness of the resin was reduced, which in turn caused frictional force to be produced between the resin and the FBG, in addition to vacuum compression, thus causing loading to the FBG.

In 2010, Takeda et al..$^{(4)}$ embedded FBG sensors into a carbon fiber reinforced polymer (CFRP) to examine the relationship between laminate thickness and internal residual strain following the curing process. Experimental results indicated that spectral distortions of the CFPR specimen following curing were caused by nonuniform axial strain on the given FBG sensor, which was ${ }^{\bar{*}}$ Corresponding author: e-mail: ccchiang@kuas.edu.tw 
attributed to the use of carbon fibers. Final calculations based on spectral data showed an inverse relationship between laminate thickness and residual strain, and that the residual strains for the laminated layers 4,8 , and 13 were $-1233,-1367$, and $-1650 \mu \varepsilon$, respectively.

In 2011, Shen ${ }^{(5)}$ embedded FBG sensors into the different laminated layers of $\left[0^{\circ}\right]_{28}$ carbon fiber epoxy composite to measure residual strain. The results indicated that the residual strain for the middle layer was $370 \mu \varepsilon$. Gelation and solidification of the composite material can also be observed when monitoring the curing process.

A literature survey reveals that most of the previous studies in this area have focused on damage evaluation and on measuring the mechanical properties of composite materials, but that they lack any consideration of residual strain monitoring after curing in different layers with different angles of $0,-45,90$, and $45^{\circ}$ isotropic laminates. The aim of the current study was to apply FBG sensors to monitor the characteristics of the curing process in a quasi-isotropic carbon fiber/epoxy composite. Four FBGs were embedded into different lamina of composite materials, after which the progress of curing as well as internal residual strain values during the curing process were measured.

\section{Materials and Methods}

\subsection{Working principle of FBG sensors}

FBG, which is also known as short-period fiber grating, possesses a grating cycle of approximately $1 \mu \mathrm{m}$. When FBG is influenced by physical quantities, the structural characteristics of the FBG are altered. Because the FBG structure is located in the core of optical fibers, it is primarily affected by temperature and strain, which cause shifts in the reflected wavelength. When subjected to tension or when placed in an environment with an increasing temperature, the reflected wavelength of the FBG is generally increased. Conversely, the reflected wavelength of the FBG is reduced when the FBG is subjected to pressure or placed in an environment with a decreasing temperature. The required physical value can be determined by incorporating the shift variation of the front and back portions of the reflective wavelength into the Bragg reflection equation as shown here: $(6,7)$

$$
\lambda=2 n_{\mathrm{eff}} \Lambda,
$$

where $\lambda$ represents the Bragg wavelength, $n_{\text {eff }}$ represents the effective refractive index, and $\Lambda$ represents the grating cycle. These values can be further developed into the following equations: ${ }^{(6)}$

$$
\begin{gathered}
\frac{\Delta \lambda}{\lambda}=\left\{1-\frac{n_{\mathrm{eff}}^{2}}{2}\left[p_{12}-v\left(p_{11}+p_{12}\right)\right]\right\} \varepsilon_{z}=\left(1-p_{\mathrm{e}}\right) \varepsilon_{z}, \\
p_{\mathrm{e}}=\frac{n_{\mathrm{eff}}^{2}}{2}\left[p_{12}-v\left(p_{11}+p_{12}\right)\right],
\end{gathered}
$$

where $p_{\mathrm{e}}$ is the elasto-optic coefficient and $p_{11}$ and $p_{12}$ represent the elasto-optic tensor with $p_{11}=$ 0.113 and $p_{12}=0.252$, respectively, $n_{\text {eff }}=1.458$, and $v=0.16$. The relationship between the FBG 
wavelength shift and axial and parallel strain are obtained in Refs. 6, 8-10. The triaxial strain is represented as

$$
\begin{gathered}
\varepsilon_{z}=\frac{1}{E \cdot \operatorname{det}|\boldsymbol{F}|}\left\{\left(1-v^{2}\right)\left[\frac{-F_{3} \Delta \lambda_{x}+F_{1} \Delta \lambda_{y}}{\lambda}\right]+\left(-v^{2}-v\right)\left[\frac{F_{4} \Delta \lambda_{x}-F_{2} \Delta \lambda_{y}}{\lambda}\right]\right\}, \\
\varepsilon_{x}=\frac{1}{E \cdot \operatorname{det}|\boldsymbol{F}|}\left\{\left(-v^{2}-v\right)\left[\frac{-F_{3} \Delta \lambda_{x}+F_{1} \Delta \lambda_{y}}{\lambda}\right]+\left(1-v^{2}\right)\left[\frac{F_{4} \Delta \lambda_{x}-F_{2} \Delta \lambda_{y}}{\lambda}\right]\right\}, \\
\varepsilon_{y}=0,
\end{gathered}
$$

where

$$
\begin{gathered}
F_{1}=\frac{-v^{2}-v}{E}+\frac{n_{\mathrm{eff}}^{2}\left[p_{11}\left(v^{2}-1\right)+p_{12}\left(v^{2}-v\right)\right]}{2 E}, \\
F_{2}=\frac{1-v^{2}}{E}+\frac{n_{\mathrm{eff}}^{2}\left[p_{11}\left(v^{2}+v\right)+p_{12}\left(v^{2}-1\right)\right]}{2 E}, \\
F_{3}=\frac{-v^{2}-v}{E}+\frac{n_{\mathrm{eff}}^{2} p_{12}\left(2 v^{2}+v-1\right)}{2 E} \\
F_{4}=\frac{1-v^{2}}{E}+\frac{n_{\mathrm{eff}}^{2} p_{12}\left(2 v^{2}+v-1\right)}{2 E} \\
\boldsymbol{F}=\left[\begin{array}{ll}
F_{1} & F_{2} \\
F_{3} & F_{4}
\end{array}\right]
\end{gathered}
$$

In the above equations, $v$ represents Poisson's ratio, and $v=0.16$. The term $E$ represents the modulus of elasticity, $n_{\text {eff }}$ represents the effective refractive index of the optical fiber, and $p_{11}$ and $p_{12}$ represent the elasto-optic tensor with $p_{11}=0.113$ and $p_{12}=0.252$, respectively. The terms $\Delta \lambda_{x}$ and $\Delta \lambda_{y}$ represent the wavelength shifts in directions $x$ and $y$, respectively, and $\lambda$ represents the Bragg wavelength.

\subsection{Experimental}

Temperature-corrected FBG sensors $[5 \mathrm{~mm}$ length of the sensing part and the full width half maximum (FWHM) of the FBG of about $0.175 \mathrm{~nm}$ ] were embedded into prepreg materials with different layers according to wavelength, from small to large. The layer orders and locations were $\left[45^{\circ} / 90^{\circ} /-45^{\circ} / 0^{\circ} / 45^{\circ} / 90^{\circ} /-45^{\circ} / 0^{\circ} /[\mathrm{S} 1] / 0^{\circ} /-45^{\circ} /[\mathrm{S} 2] / 90^{\circ} / 45^{\circ} /[\mathrm{S} 3] / 0^{\circ} /-45^{\circ} /[\mathrm{S} 4] / 90^{\circ} / 45^{\circ}\right]$.

The prepreg laminate composite consisted of 16 layers and was finished using a curing process that involved heating and pressurizing inside a mold. First, a teflon fabric was laid in the mold, followed by a prepreg material, another layer of teflon fabric, and then a diaphragm and an O-ring. Next, the mold was locked and sealed. The upper and lower molds were connected by an air 
compressor and vacuum pump lines and laid into a thermal chamber to thermoform. Figure 1 shows the experimental setup for the FBG monitoring of composite curing.

\section{Results and Discussion}

As shown in Fig. 2, some slight wavelength shifts occurred during the curing process as the temperature rose. The spectrum widths narrowed as the FBG sensor locations moved toward the interior. When the temperature was between 70 and $75^{\circ} \mathrm{C}$, the wavelength-temperature curves in the spectra are downward and nonlinear for sensors S1 and S2. The wavelength-temperature curves in the spectra change upward at $105{ }^{\circ} \mathrm{C}$ for sensor $\mathrm{S} 1$ and at $100{ }^{\circ} \mathrm{C}$ for sensor $\mathrm{S} 2$. There were also wavelength shifts towards longer wavelengths. The wavelength shifts were more apparent in FBG sensor S1, the innermost sensor, at temperatures below $60{ }^{\circ} \mathrm{C}$, in sensor $\mathrm{S} 2$ at temperatures below $55^{\circ} \mathrm{C}$, and in sensors $\mathrm{S} 3$ and $\mathrm{S} 4$, the outermost sensors, at temperatures below $45^{\circ} \mathrm{C}$.

Figure 3 illustrates the S1-S4 wavelengths of peaks before and after curing. After curing, the wavelengths shifted toward shorter wavelengths, which may be due to compressive residual strain, as proposed in photoelasticity theory. A single peak was split into two peaks in the spectra of sensors S1 and S2. FBG theory suggests that birefringence may have occurred in the four FBGs due to lateral residual strain. A split into two peaks occurred in sensor S1 due to the residual strain of the isotropic laminate. It showed that the residual stress obviously existed in Fig. 3 because there was no superposition of the curves in the spectra. Sensor S1 embedded as the innermost sensor retained the highest stress and sensor S2 had the second highest stress, as shown in Table 1. The residual stress was less affected for sensors $\mathrm{S} 3$ and $\mathrm{S} 4$, because they were embedded as the outermost sensors. The axial residual stress, axial strain, lateral residual stress, and lateral strain in the FBGs were calculated via central wavelength shifts and distances between split peaks before and after curing (Table 1).

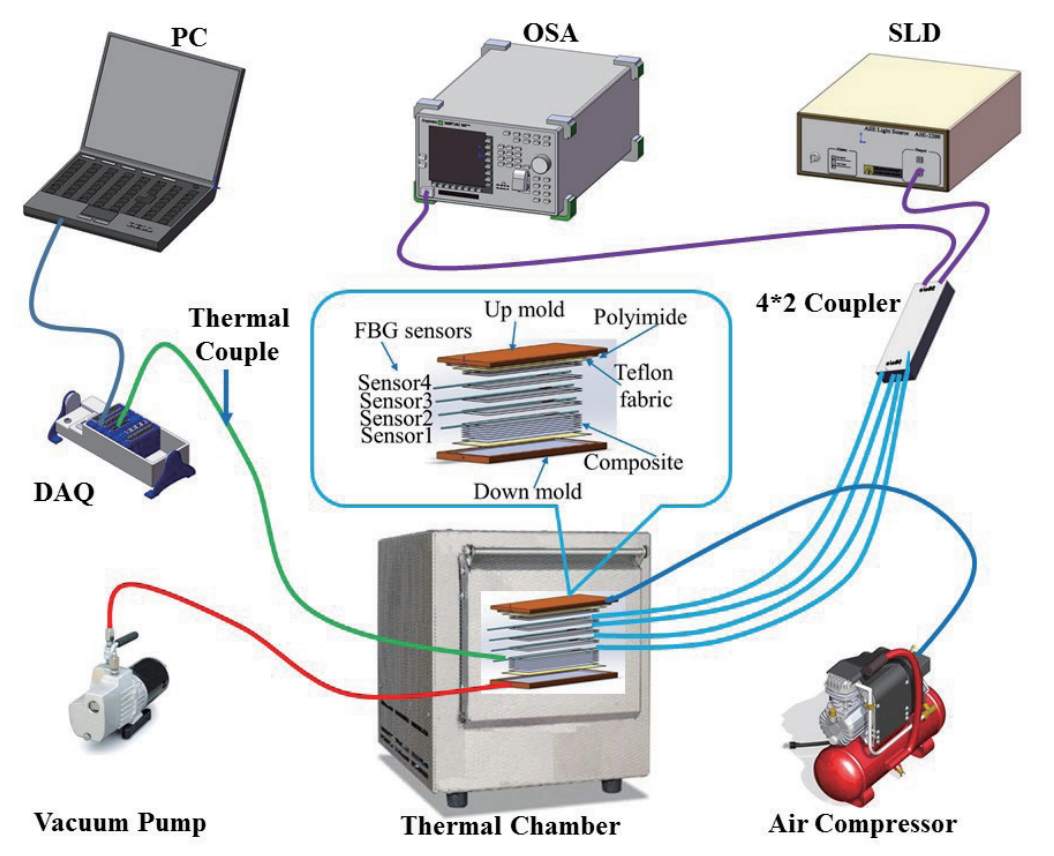

Fig. 1. (Color online) The experimental setup for FBG monitoring of composite curing. 

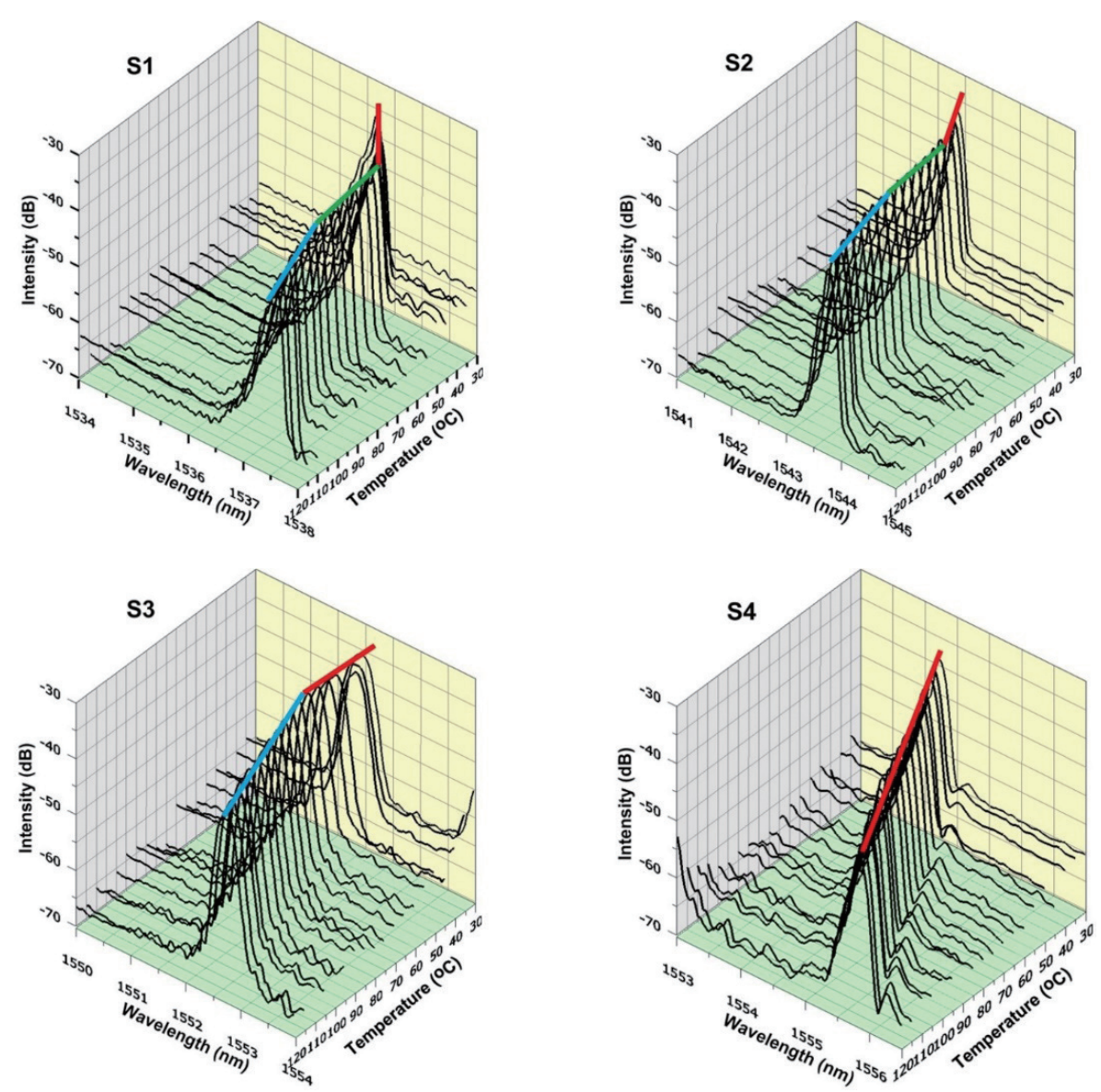

Fig. 2. (Color online) S1-S4 wavelength shifts during curing.
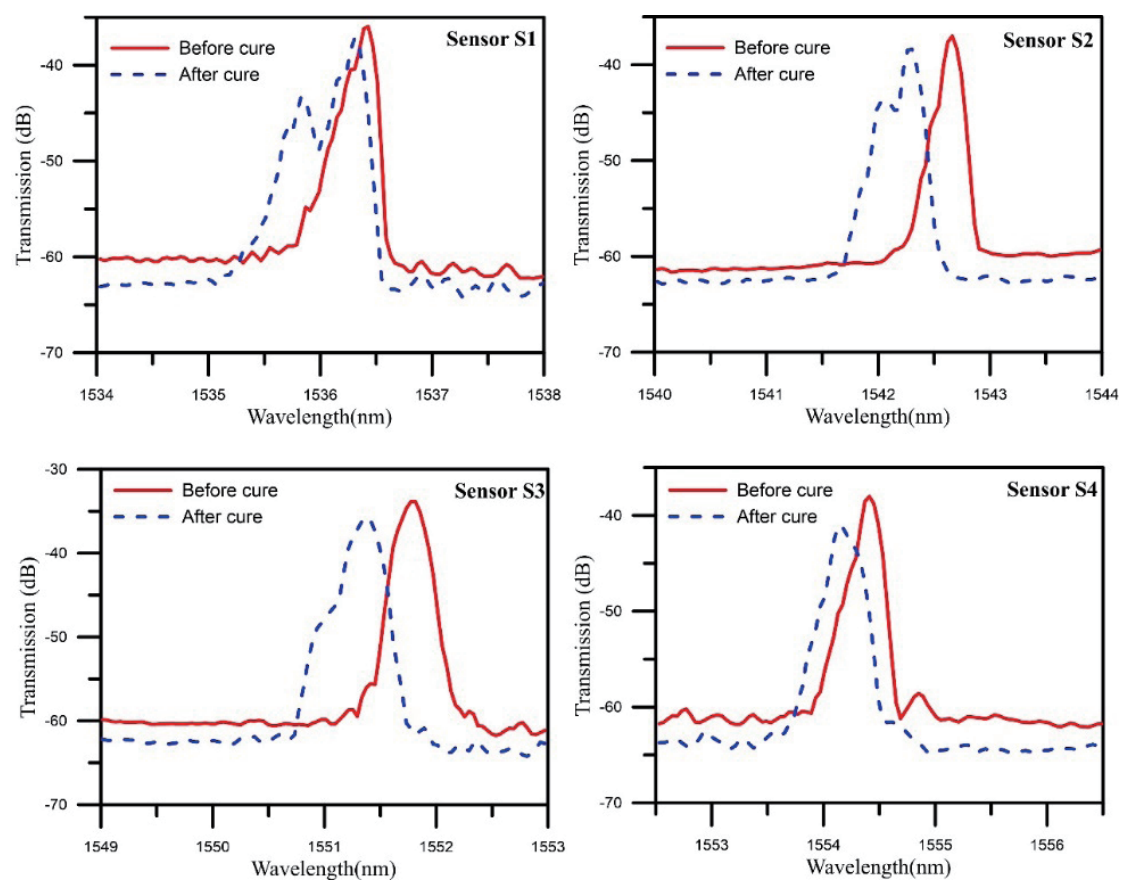

Fig. 3. (Color online) Wavelengths before and after curing. 
Table 1

S1-S4 Residual stresses and residual strains.

\begin{tabular}{|c|c|c|c|c|c|}
\hline \multicolumn{6}{|c|}{$\left[45^{\circ} / 90^{\circ} /-45^{\circ} / 0^{\circ} / 45^{\circ} / 90^{\circ} /-45^{\circ} / 0^{\circ} /[\mathrm{S} 1] / 0^{\circ} /-45^{\circ} /[\mathrm{S} 2] / 90^{\circ} / 45^{\circ} /[\mathrm{S} 3] / 0^{\circ} /-45^{\circ} /[\mathrm{S} 4] / 90^{\circ} / 45^{\circ}\right]$} \\
\hline \multicolumn{3}{|c|}{ Sensor S1 } & \multicolumn{3}{|c|}{ Sensor S2 } \\
\hline$\sigma_{z}$ & $\sigma_{x}$ & $\sigma_{y}$ & $\sigma_{z}$ & $\sigma_{x}$ & $\sigma_{y}$ \\
\hline-24.7 & 93.4 & 18.9 & -2.1 & 63.4 & 10.5 \\
\hline$\varepsilon_{z}$ & $\varepsilon_{x}$ & $\varepsilon_{y}$ & $\varepsilon_{z}$ & $\varepsilon_{x}$ & $\varepsilon_{y}$ \\
\hline-96.2 & 1234.6 & 0 & -138.5 & 877.5 & 0 \\
\hline \multicolumn{3}{|c|}{ Sensor S3 } & \multicolumn{3}{|c|}{ Sensor S4 } \\
\hline$\sigma_{z}$ & $\sigma_{x}$ & $\sigma_{y}$ & $\sigma_{z}$ & $\sigma_{x}$ & $\sigma_{y}$ \\
\hline-27 & -4.1 & -4 & -34.1 & -6.6 & -6.5 \\
\hline$\varepsilon_{z}$ & $\varepsilon_{x}$ & $\varepsilon_{y}$ & $\varepsilon_{z}$ & $\varepsilon_{x}$ & $\varepsilon_{y}$ \\
\hline-281.3 & -1.2 & 0 & -456.7 & -1.9 & 0 \\
\hline
\end{tabular}

${ }^{*}$ Stress $(\sigma)$ unit, MPa; Strain $(\varepsilon)$ unit, $\mu \varepsilon$.

\section{Conclusions}

The results showed that the residual stresses, strains, and splits were generated from the innermost to the outermost laminates $\left(0,-45,90\right.$, and $45^{\circ}$ isotropic laminates). Thus, it can be assumed that the material orientations affect the order in which stress is generated and determine the type of strain (compression or stretching).

The upper and lower laminates $\left[-45^{\circ} / 0^{\circ} /[\mathrm{S} 1] / 0^{\circ} /-45^{\circ}\right]$ of the innermost sensor S1 were protected by $0^{\circ}$ layers on either side, and the lateral stress was relatively mild. A split into two peaks also occurred in sensor S1. It was assumed that the refractive index of FBG sensor S1 changed owing to anisotropic ellipse polarization caused by isotropic laminate residual stress. The experimental results showed that the four FBG sensors embedded into isotropic carbon fiber/epoxy composite had a maximum axial residual strain of $-456.7 \mu \varepsilon$ and a maximum lateral residual strain of $1234.6 \mu \varepsilon$.

\section{Acknowledgements}

This work was supported by the Ministry of Science and Technology, Taiwan (grant number MOST 103-2221-E-151-009-MY3).

\section{References}

1 F. G. Tomasel and P. A. A. Laura: J. Sound Vibration 253 (2002) 523.

2 J. S. Leng and A. Asundi: Sens. Actuators, A 103 (2003) 330.

3 M. Mulle, F. Collombet, P. Olivier, and Y. H. Grunevald: Compos. Part A, 40 (2009) 94.

4 S. I. Takeda, J. Koyanagi, S. Utsunomiya, Y. Kinoshita, Y. Arao, and H. Kawada: Proc. SPIE 7522, Fourth International Conference on Experimental Mechanics, 75223N (15 April 2010).

5 X. Y. Shen: Adv. Mater. Res. Trans. Technol. Publ. (2011) 585.

6 M. Majumder, T. K. Gangopadhyay, A. K. Chakraborty, K. Dasgupta, and D. K. Bhattacharya: Sens. Actuators, A. 147 (2008) 150.

7 R. Kashyap: Principles of Optical Fiber Grating Sensors, 2nd ed. (Academic Press, Boston, 2009) Chap. 10. pp. 441-502.

8 J. A. Guemes and J. M. Menendez: Compos. Sci. Technol. 62 (2002) 959.

9 J. M. Menendez and J. A. Guemes: Proc. SPIE 3986 (2000) 271.

10 R. Gafsi and M. A. E. Sherif: Opt. Fiber Technol. 6 (2000) 299. 